\title{
THE TYPE OF THE MAXIMAL OPERATORS OF A CLASS OF WALSH CONVOLUTION OPERATORS
}

\author{
HE ZELIN AND DAVID MUSTARD
}

(Communicated by J. Marshall Ash)

\begin{abstract}
This paper discusses the properties of a class of $p$-adic Walsh convolution operators. The class consists of those 1-parameter sets of operators with kernels that can be represented as the $p$-adic Walsh-Fourier integral of a uniformly quasi-convex function. The paper proves that the maximal operators associated with each 1-parameter set are all of strong type $(\infty, \infty)$ and of weak type $(1,1)$.
\end{abstract}

\section{INTRODUCTION}

Properties of the Walsh-Fourier series and their various summations have been discussed often, for example, in [1, 3, 6, 8, 9, 13, 14]. In 1955 Selfridge [11] developed the Walsh transform on $L\left(\mathbb{R}^{+}\right)$(beyond that initially introduced by Fine [4]) and discussed some of its properties. Among other things, he discussed the modified Fejér mean

$$
\lim _{\rho \rightarrow \infty} \int_{0}^{\rho}\left(1-\frac{[u]}{\rho}\right) \hat{f}(u) \psi_{x}(u) d u,
$$

where $\hat{f}$ is the Walsh transform of $f,[u]$ is the greatest integer less than or equal to $u$, and $\psi_{x}$ is the $p$-adic Walsh function with index $x\left(x \in \mathbb{R}^{+}\right)$, and showed that for some integrable functions (1) does not converge a.e.

There has been very little further discussion on the properties of the various means of the inverse Walsh transform on $L\left(\mathbb{R}^{+}\right)$, but that field seems a more mathematically interesting field than that merely of the summation of WalshFourier series.

The usual definition of the Fejér mean $T_{\rho} f$ is

$$
\forall q \in[1,2], \forall f \in L^{q}\left(\mathbb{R}^{+}\right) \quad T_{\rho} f=\int_{0}^{\rho}\left(1-\frac{u}{\rho}\right) \hat{f}(u) \psi_{\bullet}(u) d u,
$$

and the integral in this can be rewritten as

$$
T_{\rho} f=f * \int_{0}^{\rho}\left(1-\frac{u}{\rho}\right) \psi_{\bullet}(u) d u,
$$

Received by the editors May 16, 1990 and, in revised form, April 1, 1991.

1991 Mathematics Subject Classification. Primary 42C10. 
where $*$ is the ( $p$-adic) Walsh convolution operation. In this paper we adopt $\left(2^{\prime}\right)$ as the defining integral because it is well defined for $f \in L^{q}\left(\mathbb{R}^{+}\right)$, not only for $q \in[1,2]$ but also for $q \in[1, \infty]$.

In this paper we shall discuss the type of the maximal operator $T$ where

$$
T f=\sup _{\rho \in \mathbb{R}^{+}}\left|T_{\rho} f\right|,
$$

and then by the representation theorem of [7] one can extend the result to more general operators. The property of convergence a.e. follows immediately.

The notation in this paper is standard and follows that (for example) of $[2,11]$.

\section{THE FIRST THEOREM}

Theorem 1. Let $T f(x)=\sup _{\rho \in \mathbb{R}^{+}}\left|f * \int_{0}^{\rho}(1-u / \rho) \psi_{x}(u) d u\right|$. Then $T$ is of strong type $(\infty, \infty)$ and of weak type $(1,1)$, and so also of strong type $(p, p)$ for $1<p<\infty$.

Proof. Our plan is to expand $u\left(u \in \mathbb{R}^{+}\right)$in a $p$-adic Walsh series and then to obtain a majorant function of $\int_{0}^{\rho}(1-u / \rho) \psi_{x}(u) d u$ from which we get that $T$ is of strong type $(\infty, \infty)$. From this base we use a Calderón-Zygmund decomposition to prove that $T$ is of weak type $(1,1)$.

First we find the $p$-adic Walsh series expansion of $x \in \mathbb{R}^{+}$(cf. [5]). Let $x \in[0,1)$ have the $p$-adic expansion

$$
x=\sum_{k=1}^{\infty} x_{k} p^{-k}, \quad x_{k} \in\{0,1,2, \ldots, p-1\} .
$$

We show that there exists a unique ordered set of $p$ numbers $\left(a_{0}, a_{1}, \ldots, a_{p-1}\right)$ such that

$$
\sum_{l=0}^{p-1} a_{l} \varphi_{k-1}^{l}(x)=x_{k},
$$

where $\varphi_{k}(x)$ are the $p$-adic Rademacher functions. On using the definition $\varphi_{k}(x)=e^{(2 \pi i / p) x_{k+1}}$, equation (5) becomes

$$
\sum_{l=0}^{p-1} a_{l} e^{(2 \pi i / p) x_{k} l}=x_{k} \quad\left(x_{k}=0,1, \ldots, p-1\right) ;
$$

that is, in detail,

$$
\begin{aligned}
a_{0}+a_{1}+\cdots+a_{p-1} & =0 \\
a_{0}+a_{1} e^{2 \pi i / p}+\cdots+a_{p-1} e^{(2 \pi i / p)(p-1)} & =1 ; \\
& \vdots \\
a_{0}+a_{1} e^{(2 \pi i / p)(p-1)}+\cdots+a_{p-1} e^{(2 \pi i / p)(p-1)^{2}} & =p-1 .
\end{aligned}
$$

This is a Vandermonde system of equations and the determinant of its coefficient matrix is

$$
\left|\begin{array}{cccc}
1 & 1 & \cdots & 1 \\
1 & e^{2 \pi i / p} & \cdots & e^{(2 \pi i / p)(p-1)} \\
\vdots & \vdots & \vdots & \vdots \\
1 & e^{(2 \pi i / p)(p-1)} & \cdots & e^{(2 \pi i / p)(p-1)^{2}}
\end{array}\right|
$$


which clearly does not vanish, and so it has a unique solution, which one easily gets as

$$
a_{0}=\frac{p-1}{2}, \quad a_{l}=\frac{1}{e^{-(2 \pi i / p) l}-1} \quad(l=1,2, \ldots, p-1) .
$$

Substituting (5) into (4), one gets the $p$-adic Walsh series expansion of $x \in$ $[0,1)$

$$
\begin{aligned}
x & =\sum_{k=1}^{\infty} \sum_{l=0}^{p-1} a_{l} \varphi_{k-1}^{l}(x) p^{-k}=\sum_{k=1}^{\infty} \sum_{l=0}^{p-1} a_{l} \psi_{l p^{k-1}}(x) p^{-k} \\
& =\sum_{k=0}^{\infty} \sum_{l=0}^{p-1} a_{l} \psi_{l p^{k}}(x) p^{-k-1} \quad(x \in[0,1)) .
\end{aligned}
$$

For $x \in\left[0, p^{n}\right)$, one has $x / p^{n} \in[0,1)$ so

$$
\frac{x}{p^{n}}=\sum_{k=0}^{\infty} \sum_{l=0}^{p-1} a_{l} \psi_{l p^{k}}\left(\frac{x}{p^{n}}\right) p^{-k-1} ;
$$

that is,

$$
x=\sum_{k=0}^{\infty} \sum_{l=0}^{p-1} a_{l} \psi_{l p^{k-n}}(x) p^{-k-1+n}
$$

that is,

$$
x=\sum_{k=-n}^{\infty} \sum_{l=0}^{p-1} a_{l} \psi_{l p^{k}}(x) p^{-k-1},
$$

the corresponding expansion for $x \in\left[0, p^{n}\right)$. Since $x$ is real, one also has

$$
x=\sum_{k=-n}^{\infty} \sum_{l=0}^{p-1} \bar{a}_{l} \bar{\psi}_{l p^{k}}(x) p^{-k-1} \quad\left(x \in\left[0, p^{n}\right)\right) .
$$

Now if $x \in \mathbb{R}^{+}$then there is an $m \in \mathbb{N}$ such that $x \in\left[0, p^{m}\right)$, and then

$$
\begin{aligned}
\sum_{k=-\infty}^{-m-1} \sum_{l=0}^{p-1} a_{l} \psi_{l p^{k}}(x) p^{-k-1} & =\sum_{k=-\infty}^{-m-1} \sum_{l=0}^{p-1} a_{l} \psi_{l}\left(p^{k} x\right) p^{-k-1} \\
& =\sum_{k=-\infty}^{-m-1}\left(\sum_{l=0}^{p-1} a_{l}\right) p^{-k-1}=0,
\end{aligned}
$$

and so we have the expansion for $x \in \mathbb{R}^{+}$as

$$
x=\sum_{k=-\infty}^{\infty} \sum_{l=0}^{p-1} a_{l} \psi_{l p^{k}}(x) p^{-k-1} \quad\left(x \in \mathbb{R}^{+}\right)
$$

and

$$
x=\sum_{k=-\infty}^{\infty} \sum_{l=0}^{p-1} \bar{a}_{l} \bar{\psi}_{l p^{k}}(x) p^{-k-1} \quad\left(x \in \mathbb{R}^{+}\right)
$$


Having found the Walsh series expansion of $u$, we can get the majorant function of $\int_{0}^{\rho}(1-u / \rho) \psi_{t}(u) d u$. Let

$$
\rho=\sum_{q=1}^{\infty} \alpha_{q} p^{n_{q}},
$$

where $n_{1}>n_{2}>\cdots>n_{q}>\cdots \quad\left(n_{q} \in \mathbb{Z}\right)$ and $\alpha_{q} \in\{1,2, \ldots, p-1\}$. Then

$$
\begin{aligned}
& \int_{0}^{\rho}\left(1-\frac{u}{\rho}\right) \psi_{t}(u) d u \\
& \quad=\left(\int_{0}^{\alpha_{1} p^{n_{1}}}+\int_{\alpha_{1} p^{n_{1}}}^{\alpha_{1} p^{n_{1}}+\alpha_{2} p^{n_{2}}}+\cdots+\int_{\alpha_{1} p^{n_{1}+\cdots+\alpha_{q-1}}}^{\alpha_{1} p^{n_{1}}+\cdots+\alpha_{q} p^{n_{q}}}+\cdots\right) \\
& \quad \times\left(1-\frac{u}{\rho}\right) \psi_{t}(u) d u,
\end{aligned}
$$

and we now estimate each term of the last expression. By (12)

$$
\begin{aligned}
\int_{0}^{p^{n_{1}}} & \left(1-\frac{u}{\rho}\right) \psi_{t}(u) d u \\
= & p^{n_{1}} \chi_{\left[0, p^{\left.-n_{1}\right)}\right.}(t)-\frac{1}{\rho} \sum_{k=-n_{1}}^{\infty} \bar{a}_{l} p^{-k-1} \int_{0}^{p^{n_{1}}} \bar{\psi}_{l p^{k}}(u) \psi_{t}(u) d u \\
= & p^{n_{1}} \chi_{\left[0, p^{\left.-n_{1}\right)}\right.}(t)-\frac{1}{\rho} \sum_{k=-n_{1}}^{\infty} \sum_{l=0}^{p-1} \bar{a}_{l} p^{-k-1+n_{1}} \int_{0}^{1} \bar{\psi}_{l p^{k+n_{1}}}(u) \psi_{t p^{n_{1}}}(u) d u \\
= & p^{n_{1}} \chi_{\left[0, p^{\left.-n_{1}\right)}\right.}(t)-\frac{1}{\rho} \sum_{k=-n_{1}}^{\infty} \sum_{l=0}^{p-1} \bar{a}_{l} p^{-k-1+n_{1}} \chi_{\left[l p^{k}, l p^{k}+p^{\left.-n_{1}\right)}\right.}(t),
\end{aligned}
$$

where $\chi_{S}(t)$ denotes the characteristic function of the set $S$. So

$$
\begin{aligned}
\int_{0}^{\alpha_{1} p^{n_{1}}}\left(1-\frac{u}{\rho}\right) \psi_{t}(u) d u & =\sum_{r=0}^{\alpha_{1}-1} \int_{r p^{n_{1}}}^{(r+1) p^{n_{1}}}\left(1-\frac{u}{\rho}\right) \psi_{t}(u) d u \\
& =\sum_{r=0}^{\alpha_{1}-1} \int_{0}^{p^{n_{1}}}\left(1-\frac{r p^{n_{1}}}{\rho}-\frac{u}{\rho}\right) \psi_{t}\left(u+r p^{n_{1}}\right) d u .
\end{aligned}
$$

Because $0<u<p^{n_{1}}$, it follows that $\psi_{t}\left(u+r p^{n_{1}}\right)=\psi_{t}\left(u \oplus r p^{n_{1}}\right)=\psi_{t}(u) \psi\left(r p^{n_{1}}\right)$ a.e. and so

$$
\begin{aligned}
& \sum_{r=0}^{\alpha_{1}-1} \int_{0}^{p^{n_{1}}}\left(1-\frac{r p^{n_{1}}}{\rho}-\frac{u}{\rho}\right) \psi_{t}\left(u+r p^{n_{1}}\right) d u \\
& =\sum_{r=0}^{\alpha_{1}-1}\left[\int_{0}^{p^{n_{1}}}\left(1-\frac{r p^{n_{1}}}{\rho}\right) \psi_{t}(u) d u-\frac{1}{\rho} \int_{0}^{p^{n_{1}}} u \psi_{t}(u) d u\right] \psi_{t}\left(r p^{n_{1}}\right) \\
& =\frac{1}{\rho} \sum_{r=0}^{\alpha_{1}-1}\left[\left(\rho-r p^{n_{1}}\right) p^{n_{1}} \chi_{\left[0, p^{\left.-n_{1}\right)}\right.}(t)-\sum_{k=-n_{1}}^{\infty} \sum_{l=0}^{p-1} \bar{a}_{l} p^{-k-1+n_{1}} \chi_{\left[l p^{k}, l p^{k}+p^{\left.-n_{1}\right)}\right.}(t)\right] \\
& \times \psi_{t}\left(r p^{n_{1}}\right) .
\end{aligned}
$$




\section{Similarly}

$$
\begin{aligned}
& \int_{\alpha_{1} p^{n_{1}}}^{\alpha_{1} p^{n_{1}}+\alpha_{2} p^{n_{2}}}\left(1-\frac{u}{\rho}\right) \psi_{t}(u) d u \\
& (17) \quad=\int_{0}^{\alpha_{2} p^{n_{2}}}\left(1-\frac{u+\alpha_{1} p^{n_{1}}}{\rho}\right) \psi_{t}\left(u+\alpha_{1} p^{n_{1}}\right) d u \\
& =\frac{1}{\rho} \sum_{r=0}^{\alpha_{2}-1}\left[\left(\rho-\alpha_{1} p^{n_{1}}+r p^{n_{2}}\right) p^{n_{2}} \chi_{\left[0, p^{\left.-n_{2}\right)}\right.}(t)\right. \\
& \left.\quad-\sum_{k=-n_{2}}^{\infty} \sum_{l=0}^{p-1} \bar{a}_{l} p^{-k-1+n_{2}} \chi_{\left[l p^{k}, l p^{k}+p^{\left.-n_{2}\right)}\right.}(t)\right] \psi_{t}\left(\alpha_{1} p^{n_{1}}+r p^{n_{2}}\right)
\end{aligned}
$$

and

$$
\begin{gathered}
\int_{\alpha_{1} p^{n_{1}}+\cdots+\alpha_{q-1} p^{n_{q-1}}}^{\alpha_{1} p^{n_{1}}+\cdots+\alpha_{q-1} p^{n_{q-1}}+\alpha_{q} p_{q}^{n}}\left(1-\frac{u}{\rho}\right) \psi_{t}(u) d u \\
=\frac{1}{\rho} \sum_{r=0}^{\rho_{q-1}}\left[\left(\rho-\alpha_{1} p^{n_{1}}-\cdots-\alpha_{q-1} p^{n_{q-1}}-r p^{n_{q}}\right) p^{n_{q}} \chi_{\left[0, p^{\left.-n_{q}\right)}\right.}(t)\right. \\
\left.-\sum_{k=-n_{q}}^{\infty} \sum_{l=0}^{p-1} \bar{a}_{l} p^{-k-1+n_{q}} \chi_{\left[l p^{k}, l p^{k}+p^{-n_{q}}\right)}(t)\right] \\
\times \psi_{t}\left(\alpha_{1} p^{n_{q}}+\cdots+\alpha_{q-1} p^{n_{q-1}}+r p^{n_{q}}\right) .
\end{gathered}
$$

So by (16)-(18) we have

$$
\left|\int_{0}^{\rho}\left(1-\frac{u}{\rho}\right) \psi_{t}(u) d u\right| \leq \frac{A}{\rho} \sum_{j=1}^{\infty} \sum_{k=-n_{j}}^{\infty} \sum_{l=0}^{p-1} p^{-k-1+n_{j}} \chi_{\left[l p^{k}, l p^{k}+p^{-n_{j}}\right)}(t),
$$

where $A$ is a constant. Let

$$
\begin{gathered}
K_{\rho}^{*}(t)=\frac{A}{\rho} \sum_{j=1}^{\infty} \sum_{k=-n_{j}}^{\infty} \sum_{l=0}^{p-1} p^{-k-1+n_{j}} \chi_{\left[l p^{k}, l p^{k}+p^{\left.-n_{j}\right)}\right.}(t), \\
T^{*} f=\sup _{\rho \in \mathbb{R}^{+}}\left|f * K_{\rho}^{*}\right|,
\end{gathered}
$$

and

$$
M f=\sup _{\rho \in \mathbb{R}^{+}}|f| * K_{\rho}^{*} .
$$

\section{Clearly}

$$
(T f)(x) \leq(M f)(x) \text { and }\left(T^{*} f\right)(x) \leq(M f)(x)
$$

By (19)

$$
\begin{aligned}
\|M f\|_{\infty} & \leq \sup _{\rho}\left\|K_{\rho}^{*}\right\|_{1}\|f\|_{\infty} \leq \sup _{\rho} \frac{A}{\rho} \sum_{j=1}^{\infty} \sum_{k=-n_{j}}^{\infty} p^{-k}\|f\|_{\infty} \\
& \leq\|f\|_{\infty} \sup _{\rho} \frac{A}{\rho} \sum_{j=1}^{\infty} \frac{p^{n_{j}}}{1-\frac{1}{p}} \leq\|f\|_{\infty} A \frac{p}{p-1}=B\|f\|_{\infty},
\end{aligned}
$$


where $B=p A /(p-1)$; so $M$ is of strong type $(\infty, \infty)$ (and, of course, $T$ and $T^{*}$ are too).

In what follows we prove $T$ is of weak type $(1,1)$. Clearly it is enough to prove that $M$ is of weak type $(1,1)$.

By the Calderón-Zygmund method (cf. [12] for the dyadic case-it is similar to the $p$-adic case) we have that for every $\lambda>0$ there exists a sequence $\left\{I_{m}\right\}$ of open disjoint $p$-adic subintervals such that

$$
|f(x)|=g(x)+b(x),
$$

where

$$
\|g\|_{\infty} \leq p \lambda
$$

and

$$
b(x)=\sum b_{m}(x)
$$

here

$$
\begin{gathered}
b_{m}(x)=0 \text { if } x \notin I_{m} \text { and } \int_{I_{m}} b_{m}(x) d x=0, \\
\|b\|_{1} \leq 2\|f\|_{1},
\end{gathered}
$$

and

$$
|\Omega|=\left|\bigcup I_{m}\right| \leq \frac{1}{\lambda}\|f\|_{1}
$$

Because

$$
\begin{aligned}
M f & =\sup _{\rho}|f| * K_{\rho}^{*}=\sup _{\rho}(g+b) * K_{\rho}^{*} \\
& \leq \sup \left|g * K_{\rho}^{*}\right|+\sup _{\rho}\left|b * K_{\rho}^{*}\right|=T^{*} g+T^{*} b
\end{aligned}
$$

and $T^{*}$ is of strong type $(\infty, \infty)$ and $|g(x)| \leq p \lambda$ a.e., it follows that

$$
\left\|T^{*} g\right\|_{\infty} \leq B\|g\|_{\infty} \leq C \lambda
$$

where $C=B p$.

By (29), (30), (28), and (26),

$$
\begin{aligned}
\mid\{x & \left.\in \mathbb{R}^{+} \mid(M f)(x)>(1+C) \lambda\right\}|\leq|\left\{x \in \mathbb{R}^{+} \mid\left(T^{*} b\right)(x)>\lambda\right\} \mid \\
& \leq|\Omega|+\left|\Omega^{c} \cap\left\{x \in \mathbb{R}^{+} \mid\left(T^{*} b\right)(x)>\lambda\right\}\right| \\
& \leq \frac{1}{\lambda}\|f\|_{1}+\frac{1}{\lambda} \int_{\Omega^{c}}\left(T^{*} b\right)(x) d x \leq \frac{1}{\lambda}\|f\|_{1}+\frac{1}{\lambda} \sum_{m} \int_{I_{m}^{c}}\left(T^{*} b_{m}\right)(x) d x,
\end{aligned}
$$

where $A^{c}$ denotes the complement in $\mathbb{R}^{+}$of $A$.

Now we estimate $\int_{I_{m}^{c}}\left(T^{*} b_{m}\right)(x) d x=\int_{I_{m}^{c}} \sup _{\rho}\left|b_{m} * K_{\rho}^{*}(x)\right| d x$. Notice that in this integral $x \notin I_{m}$ and

$$
b_{m} * K_{\rho}^{*}(x)=\int_{0}^{\infty} b_{m}(t) \frac{A}{\rho} \sum_{j=1}^{\infty} \sum_{k=-n_{j}}^{\infty} \sum_{l=0}^{p-1} p^{-k-1+n_{j}} \chi_{\left[l p^{k}, l p^{k}+p^{-n_{j}}\right)}(x \ominus t) d t .
$$

Let $J\left(l, k, n_{j}, x\right)$ denote the support of $\chi_{\left[l p^{k}, l p^{k}+p^{-n_{j}}\right)}(x \ominus t)$ considered as a function of $t$. It is a $p$-adic interval with length $p^{-n_{j}}$ and including $x \ominus l p^{k}$. 
There are two cases to consider.

Case 1. $\rho \leq 1 /\left|I_{m}\right|$. Here $p^{n_{j}} \leq 1 /\left|I_{m}\right| \quad(j=1,2, \ldots)$, so $p^{-n_{j}} \geq\left|I_{m}\right|$. Then for each $l \in\{0,1, \ldots, p-1\}, j=1,2 \ldots$, and $k \geq-n_{j}, I_{m}$ (the support of $\left.b_{m}(t)\right) \subset J\left(l, k, n_{j}, x\right)$ or $I_{m} \cap J\left(l, k, n_{j}, x\right)=\varnothing$, so $b_{m} * K_{\rho}^{*}(x)=0$.

Case 2. $\rho>1 /\left|I_{m}\right|$. Let the integers $j_{0}$ and $k_{0}$ be such that $p^{-n_{j_{0}+1}} \geq\left|I_{m}\right|>$ $p^{-n_{j_{0}}}$ and $p^{k_{0}+1} \geq\left|I_{m}\right|>p^{k_{0}}$. Then, by the proof of Case 1 , we see that

$$
b_{m} * K_{\rho}^{*}(x)=\int_{0}^{\infty} b_{m}(t) \frac{A}{\rho} \sum_{j=1}^{j_{0}} \sum_{k=-n_{j}}^{\infty} \sum_{l=0}^{p-1} p^{-k-1+n_{j}} \chi_{\left[l p^{k}, l p^{k}+p^{-n_{j}}\right)}(x \ominus t) d t .
$$

Because $x \notin I_{m}$ and for $k<k_{0}, x \ominus l p^{k} \notin I_{m}$, it follows that $J\left(l, k, n_{j}, x\right)$, which is a $p$-adic interval including $x \ominus l p^{k}$ with length $p^{-n_{j}} \leq$ $p^{-n_{j_{0}}}<\left|I_{m}\right|$, is disjoint from $I_{m}$, and it is easy to see that $-n_{j_{0}} \leq k_{0}$,

$$
\begin{aligned}
\left|b_{m} * K^{*} \rho(x)\right| & =\left|\int_{0}^{\infty} b_{m}(t) \frac{A}{\rho} \sum_{j=1}^{j_{0}} \sum_{k=k_{0}}^{\infty} \sum_{l=0}^{p-1} p^{-k-1+n_{j}} \chi_{\left[l p^{k}, l p^{k}+p^{-n_{j}}\right)}(x \ominus t) d t\right| \\
& \leq A \int_{0}^{\infty}\left|b_{m}(t)\right| \sum_{k=k_{0}}^{\infty} \sum_{l=0}^{p-1} p^{-k-1} \chi_{\left[l p^{k}, l p^{k}+p^{\left.k_{0}\right)}\right.}(x \ominus t) \frac{1}{\rho} \sum_{j=1}^{j_{0}} p^{n_{j}} d t \\
& \leq A \int_{0}^{\infty}\left|b_{m}(t)\right| \sum_{k=k_{0}}^{\infty} \sum_{l=0}^{p-1} p^{-k-1} \chi_{\left[l p^{k}, l p^{k}+p^{\left.k_{0}\right)}\right.}(x \ominus t) d t .
\end{aligned}
$$

Therefore

$$
\begin{aligned}
\left(T^{*} b_{m}\right)(x) & =\sup _{\rho \in \mathbb{R}^{+}}\left|b_{m} * K_{\rho}^{*}(x)\right| \\
& \leq A \int_{0}^{\infty}\left|b_{m}(t)\right| \sum_{k=k_{0}}^{\infty} \sum_{l=0}^{p-1} p^{-k-1} \chi_{\left[l p^{k}, l p^{k}+p^{k} 0\right]}(x \ominus t) d t \quad\left(x \notin I_{m}\right),
\end{aligned}
$$

and so

$$
\begin{aligned}
\int_{I_{m}^{c}}\left(T^{*} b_{m}\right)(x) d x & \leq A \int_{0}^{\infty}\left[\left|b_{m}(t)\right| \sum_{k=k_{0}}^{\infty} \sum_{l=0}^{p-1} p^{-k-1} \int_{0}^{\infty} \chi_{\left[l p^{k}, l p^{k}+p^{\left.k_{0}\right)}\right.}(x \ominus t) d x\right] d t \\
& =A \int_{0}^{\infty}\left|b_{m}(t)\right| \sum_{k=k_{0}}^{\infty} p^{-k+k_{0}} d t=\frac{A p}{p-1}\left\|b_{m}\right\|_{1} \\
& =B\left\|b_{m}\right\|_{1},
\end{aligned}
$$

where $B=A p /(p-1)$. By (31) and (32) we have

$$
\left|\left\{x \in \mathbb{R}^{+} \mid(M f)(x)>(1+C) \lambda\right\}\right| \leq \frac{1}{\lambda}\|f\|_{1}+\frac{B}{\lambda}\|b\|_{1} \leq \frac{1+2 B}{\lambda}\|f\|_{1},
$$


so

$$
\left|\left\{x \in \mathbb{R}^{+} \mid(M f)(x)>\lambda\right\}\right| \leq \frac{(1+2 B)(1+C)}{\lambda}\|f\|_{1}
$$

This completes the proof.

\section{THE SECOND THEOREM}

Having proved Theorem 1, we can get the same properties for some general operators by the results of [7].

We call $g_{\rho}(u) \quad\left(u \in \mathbb{R}^{+}\right)$"uniformly quasi-convex" if and only if for each $\rho>0, g_{\rho}(u) \in A C_{\text {loc }}\left(\mathbb{R}^{+}\right), g_{\rho}^{\prime}(u) \in B V_{\text {loc }}\left(\mathbb{R}^{+}\right)$, and there exists a constant $C$ independent of $\rho$ such that $\int_{0}^{\infty} u\left|d g_{\rho}^{\prime}(u)\right|<C$. For example, if $g(u)$ is quasi-convex then $g(u / \rho)$ is uniformly quasi-convex.

Theorem 2. If $K_{\rho}(t)=\int_{0}^{\infty} g_{\rho}(u) \psi_{t}(u) d u$ where $g_{\rho}(u)$ is uniformly quasi-convex and $g_{\rho}(\infty)=0$ then $T$, where $T f=\sup _{\rho \in \mathbb{R}^{+}}\left|f * K_{\rho}\right|$, is of strong type $(\infty, \infty)$ and of weak type $(1,1)$.

Proof. By [7] $K_{\rho}(t)=\int_{0}^{\infty} g_{\rho}(u) \psi_{t}(u) d u \in L\left(\mathbb{R}^{+}\right)$so $f \rightarrow f * K_{\rho}$ is an operator from $L^{q}\left(\mathbb{R}^{+}\right)$to $L^{q}\left(\mathbb{R}^{+}\right)$for each $q \in[1, \infty]$. By (2) in [7]

$$
\begin{aligned}
\sup _{\rho \in \mathbb{R}^{+}}\left|f * \int_{0}^{\infty} g_{\rho}(u) \psi_{t}(u) d u\right| & =\sup _{\rho \in \mathbb{R}^{+}}\left|f * \int_{0}^{\infty} u d g_{\rho}^{\prime}(u) \int_{0}^{u}\left(1-\frac{v}{u}\right) \psi_{t}(v) d v\right| \\
& =\sup _{\rho \in \mathbb{R}^{+}}\left|\int_{0}^{\infty} u d g_{\rho}^{\prime}(u)\left[f * \int_{0}^{u}\left(1-\frac{v}{u}\right) \psi_{t}(v) d v\right]\right| \\
& \leq C \sup _{\rho \in \mathbb{R}^{+}}\left|f * \int_{0}^{u}\left(1-\frac{v}{u}\right) \psi_{t}(v) d v\right|
\end{aligned}
$$

where $C=\sup _{\rho \in \mathbb{R}^{+}} \int_{0}^{\infty} u\left|d g_{\rho}^{\prime}(u)\right|$.

By Theorem 1 we get that $T$ is of strong type $(\infty, \infty)$ and of weak type $(1,1)$.

\section{ACKNOWLEDGMENT}

The first author is grateful to the University of Nanjing for study leave and to the School of Mathematics at the University of New South Wales for a visiting Fellowship during 1988 and 1989.

\section{REFERENCES}

1. S. L. Blumin, On the linear summation method for Fourier series with respect to a multiplicative system, Sibirsk Mat. Z. 9 (1968), no. 2, 449-455. (Russian)

2. P. L. Butzer and R. J. Nessel, Fourier analysis and approximation, vol. I, Birkhäuser, Basel, and Academic Press, New York, 1971.

3. N. J. Fine, Cesàro summability of Walsh-Fourier series, Proc. Nat. Acad. Sci. U.S.A. 41 (1955), 588-591.

4. N. J. Fine, On the Walsh functions, Trans. Amer. Math. Soc. 65 (1949), 372-414.

5. He Zelin, Notes on Walsh-approximation, J. Nanjing Univ. 4 (1981), 409-418.

6. __ General linear summation of Vilenkin-Fourier series, Acta Math. Hungar. 46 (1985), 285-295.

7. Univ. Math. Biquarterly 1 (1987), 15-20. 
8. J. Pál and P. Simon, On a generalization of the concept of derivative, Acta Math. Acad. Sci. Hungar. 29 (1977), 155-164.

9. F. Schipp, Uber Gewissen Maximaloperatoren, Ann. Univ. Sci. Budapest Sect. Math. 18 (1975), 189-195.

10. W. R. Wade, Recent developments in the theory of Walsh series, Internat. J. Math. Sci. 5 (1982), 625-673.

11. R. G. Selfridge, Generalized Walsh transform, Pacific J. Math. 5 (1955), 451-480.

12. E. M. Stein, Singular integrals and differentiability properties of functions, Princeton Univ. Press, Princeton, NJ, 1970.

13. S. Yano, Cesàro summability of Walsh-Fourier series, Tôhoku Math. J. 9 (1957), 267-272.

14. Young Wo Song, Mean convergence of generalized Walsh-Fourier series, Trans. Amer. Math. Soc. 218 (1976), 311-320.

Department of Mathematics, University of Nanjing, Nanjing, People's Republic of CHINA

School of Mathematics, University of New South Wales, P.O. Box 1, Kensington, New South Wales, Australia 2033 